\title{
Novas evidências de causalidade entre sistema financeiro e crescimento econômico no Brasil usando séries de tempo no domínio da frequência
}

New evidence on the causality between financial system and economic growth in Brazil using time series in the frequency domain

\author{
Bruno de Paula Rocha(1) \\ Igor Viveiros de Souza (2) \\ (1) Universidade Federal do $\mathrm{ABC}$ \\ (2) Universidade Federal de Minas Gerais
}

\begin{abstract}
This paper aims to present causality tests between financial system and economic growth in the frequency domain for Brazil. This approach allows to evaluate nonlinearities in the direction of the causality from short to longer terms. We employ Brazilian time series for GDP per capita and banking credit between 1960 and 2010. The results show variation in causality tests depending on the frequency of the cycles considered. In particular, there is evidence of that the financial system is a causal factor for longterm economic growth in Brazil.
\end{abstract}

\section{Keywords}

economic growth; financial system; time series in frequency domain.

JEL Codes O16; G18; C32.

\section{Resumo}

Este trabalho tem como objetivo apresentar testes de causalidade entre sistema financeiro e crescimento econômico no domínio da frequência, para séries temporais brasileiras. Esta abordagem permite não-linearidades associadas a mudanças de direção na causalidade do curto para o longo prazo. Para avaliar esta relação no Brasil, o artigo emprega séries temporais para o PIB per capita e de crédito bancário entre 1960 e 2010. Os resultados mostram variação nos testes de causalidade dependendo da frequência dos ciclos considerados, havendo evidências de que o sistema financeiro seja um fator causal para o crescimento econômico no Brasil, mas apenas no longo prazo.

\section{Palavras-chave}

crescimento econômico; sistema financeiro; séries de tempo no dominio da frequência.

Códigos JEL O16; G18; C32. 


\section{Introdução}

O estudo da relação entre sistema financeiro e crescimento econômico é um tema tradicional na literatura econômica. Desde trabalhos seminais como Gurley e Shaw (1955) e Goldsmith (1969), é grande a lista de contribuições que tentam mostrar a relação entre sistema financeiro e crescimento econômico. ${ }^{1}$

Mais recentemente, uma série de estudos tem enfatizado a importância do sistema financeiro como fator causal na promoção de crescimento econômico (Bernanke, 1983; King; Levine, 1993a e 1993b; Beck; Levine; Loayza, 2000; Levine; Loayza; Beck, 2000; Levine, 2005; Calderón; Liu, 2002).

Esta relação de causalidade, contudo, é fonte de controvérsia. Arestis e Demetriades (1997), por exemplo, argumentam que a relação entre finanças e crescimento econômico é específica para cada país, sendo dependente de fatores locais, como a estrutura de funcionamento de seus sistemas financeiros. Com efeito, estudos com séries de tempos para amostras de países revelam certa diversidade de resultados (Demetriades; Hussein, 1996; Luintel; Khan, 1999; Odedokun, 1996).

De forma semelhante, Arcand, Berkes e Panizza (2012) identificam uma relação não-monotônica entre sistema financeiro e crescimento econômico. Segundo esses autores, os efeitos positivos para o crescimento seriam observados apenas dentro de um limite. Em países com elevado aprofundamento financeiro, não haveria relação entre sistema financeiro e crescimento econômico.

Este trabalho tem como objetivo avaliar a relação entre sistema financeiro e crescimento econômico, buscando explorar eventuais assimetrias relacionadas ao timing desta causalidade. ${ }^{2}$ Para tanto, empregamos a metodologia desenvolvida por Breitung e Candelon (2006), que permite a operacionalização de testes de causalidade para séries temporais no domínio da frequência. A vantagem do procedimento no domínio da frequência é a flexibilidade na fixação do período de ciclo para realização do teste. Com isso, não-linearidades no nexo-causal entre sistema financeiro e crescimento podem ser identificadas por meio do exame dos testes para ciclos de curto, médio ou longo prazo.

1 Ver Levine (2005) para uma revisão dos trabalhos empíricos relacionando sistema financeiro a crescimento econômico

2 King e Levine (1993a), por exemplo, argumentam que os efeitos do sistema financeiro sobre o crescimento econômico são essencialmente de longo prazo. 
Existe uma tradição nesta literatura quanto à aplicação de testes de causalidade de Granger. No presente contexto, evidências de precedência temporal de variáveis financeiras abrem novas possibilidades para o desenho de políticas voltadas para a melhoria no funcionamento destas instituições, com vistas a influenciar o ritmo de crescimento da economia. O procedimento no domínio da frequência permite um melhor entendimento da dinâmica envolvida nesta causalidade e, em artigos recentes, vem sendo empregado para séries econômicas de outros países (Ramirez, 2013; Gómez-González et al., 2014; Stolbov, 2015), mas não ainda para o Brasil.

Usando dados anuais para o Brasil, entre 1960 e 2010, reportamos variabilidade na causalidade entre sistema financeiro e crescimento econômico para diferentes frequências de ciclos. Particularmente, os testes apontam causalidade do sistema financeiro para crescimento, mas apenas nas frequências mais baixas (longo prazo), não havendo evidências de causalidade nos ciclos de curto prazo.

Além desta introdução, este artigo possui outras quatro seções. A segunda seção apresenta uma revisão da literatura teórica e empírica, buscando pontuar os pontos distintivos da metodologia empregada. Em seguida, a metodologia econométrica proposta por Breitung e Candelon (2006) é apresentada. A seção 4 traz os dados usados nas estimações e os principais resultados obtidos. Por fim, a seção 5 sumariza as principais conclusões do artigo.

\section{Sistema financeiro e crescimento econômico}

\subsection{Referencial teórico}

A relação entre a atuação do sistema financeiro e os padrões de atividade econômica tem sido objeto de extensa literatura. Desde os trabalhos pioneiros de Gurley e Shaw (1955) e Goldsmith (1969), uma correlação positiva entre estas duas dimensões econômicas tem sido reportada. ${ }^{3}$

Recentemente, vem ganhando destaque o entendimento de que o sistema financeiro pode ser visto como uma fonte geradora de crescimento econômico de longo prazo. Para tanto, de acordo com King e Levine (1993a), foi importante o surgimento de novas teorias de crescimento eco-

3 Uma descrição desta interpretação tradicional para a relação entre sistema financeiro e desenvolvimento econômico pode ser vista em King e Levine (1993a). 
nômico que ampliassem a conceituação trazida no modelo tradicional de Solow (1956). Com as novas teorias (Romer, 1986; Lucas, 1988, por exemplo) e seus tratamentos para o desenvolvimento tecnológico e de capital humano, tornou-se possível que uma maior variedade de políticas pudesse afetar o crescimento de longo prazo. ${ }^{4}$ Contudo, de acordo com King e Levine (1993a), tão importante quanto as novas abordagens para o crescimento de longo prazo foi o entendimento do papel do sistema financeiro no processo de intermediação de recursos. Em particular, a existência de firmas especializadas não só aumenta o volume de recursos de poupança captados, mas, principalmente, altera a alocação desses recursos, podendo, dessa maneira, impactar o crescimento tecnológico e a produtividade da economia (Beck; Levine; Loayza, 2000).

Podemos ilustrar este ponto com uma exemplificação feita em Levine (2005) a respeito das opções de financiamento disponíveis a um investidor. Tipicamente, para viabilizar um novo projeto, um investidor possui três alternativas para financiar seu plano de gastos. Primeiramente, o investidor poderia financiar seu projeto com recursos próprios, o que limitaria o volume de investimento à sua disponibilidade de poupança. Outra possibilidade seria o financiamento do investimento via poupança externa à firma. Essa operação, porém, além de envolver elevados custos de transação, é caracterizada por uma relação agência, em que a firma investidora é o agente detentor de informação sobre o projeto que não pode ser obtida publicamente (sem custos) pelo dono da poupança. Nessa relação, o poupador é o principal e, dessa forma, gostaria que o investidor tomasse as decisões que lhe interessassem. Tal assimetria informacional é importante para justificar a existência de instituições especializadas na intermediação financeira, que corresponderia à terceira opção para financiamento do mencionado projeto.

Dois elementos são importantes nesta descrição proposta por Levine (2005): o financiamento externo de projetos de investimento e os custos de transação e de informação associados a esta operação. Por meio de sua habilidade em reduzir os custos de transação e de informação, o sistema financeiro contribuiria para baratear o custo externo de financiamento dos projetos de investimento (Bernanke, 1983; Rajan; Zingales, 1988; Levine, 2005). Assim, a atuação do sistema financeiro pode não apenas influenciar

4 Para uma apresentação completa desses modelos, ver Barro e Sala-i-Martin (2004). 
as taxas de poupança e investimento, mas também promover maior eficiência alocativa e inovação tecnológica.

Levine (2005) traz uma abrangente revisão da literatura relacionada ao tema. Segundo esse autor, de uma forma geral, os trabalhos apontam para cinco canais por meio dos quais o sistema financeiro executaria sua função de reduzir o custo de financiamento externo, promovendo maiores taxas de crescimento econômico de longo prazo.

O primeiro canal presente na ação dos intermediários estaria nos ganhos de escala e na consequente maior capacidade de lidar com os riscos inerentes aos projetos a serem financiados. Tais riscos estão associados à liquidez dos projetos ou a choques idiossincráticos a que os investidores estão sujeitos. Levine (2005) destaca a importância de se contar com sistemas financeiros bem organizados, líquidos e com capacidade de diversificação de risco. Dessa maneira, o investimento mediado por essas instituições não se limitaria a projetos de maior liquidez e menor risco e, muitas vezes, de menor retorno.

O segundo canal apontado por Levine (2005) enfatiza o custo para os detentores de poupança em adquirir e avaliar as informações referentes a um potencial projeto a ser financiado. As informações de interesse incluem, entre outras coisas, o conhecimento das condições econômicas (correntes e esperadas) e a capacidade administrativa da firma que irá receber o investimento. Supondo, por exemplo, que o custo de obtenção dessas informações seja fixo, os intermediários financeiros poderiam diluir tais custos ao dividi-los entre os diversos indivíduos que utilizam seus serviços. Assim, o custo para a obtenção de informação seria reduzido na presença de intermediários financeiros, permitindo a ampliação das oportunidades de investimento na economia.

Também associada à maior escala de atuação das instituições financeiras está a maior capacidade em levantar recursos. Do ponto de vista de um investidor individual, a busca do volume de recursos requerido para o financiamento de um determinado projeto pode ser inviável. Como consequência, alguns projetos poderiam ser limitados a escalas economicamente ineficientes. Portanto, a atuação de intermediários financeiros especializados, mobilizando a poupança gerada na economia, pode trazer efeitos alocativos.

Outra função refere-se ao tratamento do problema informacional, característico da operação de financiamento de projetos, que torna custosa a avaliação do cumprimento do contrato e da utilização adequada dos 
recursos levantados. Tais assimetrias informacionais podem levar os detentores de poupança a racionarem seus recursos (Stiglitz; Weiss, 1981). Com isso, Levine (2005) sustenta que tais custos, associados à aquisição de informação e garantia de que os contratos sejam cumpridos, tornam economicamente desejável a presença de instituições financeiras que tenham vantagens em exercer tais controles sobre as firmas.

Por fim, temos o argumento de que a existência de intermediários financeiros pode promover a especialização na economia. Barateando os custos de transação e informação, os intermediários financeiros podem tornar melhores as condições para especialização econômica. $\bigcirc$ ganho proveniente da maior especialização é um dos resultados canônicos da Teoria Econômica, que remonta a Adam Smith.

Segundo Levine (2005), quanto mais hábil no cumprimento dessas funções, mais desenvolvido seria o sistema financeiro. Tomando como base alguma versão dos modelos de crescimento endógeno mencionados acima, é possível estabelecer ligação entre o desenvolvimento das atividades financeiras, no sentido já proposto, e o crescimento econômico de longo prazo (King; Levine, 1993a).

\subsection{Evidências empíricas para o Brasil}

A literatura aplicada para o Brasil parece suportar a importância até aqui sugerida do sistema financeiro para a determinação de maior crescimento econômico. Nesta direção, pode ser útil citarmos os trabalhos de Matos (2002) e Marques Jr. e Porto Jr. (2004), que testam a relação de causalidade entre sistema financeiro e crescimento econômico para séries de tempo brasileiras. Esses autores empregam a metodologia de causalidade de Granger (1969), que será apresentado com mais detalhes em nossa seção metodológica. Nos casos de não estacionariedade nas variáveis, os autores estendem o procedimento para considerar a causalidade no contexto de uma relação de cointegração (Johansen, 1988).

Matos (2002) emprega um conjunto de variáveis para mensurar o desenvolvimento financeiro: a diferença nos agregados monetários $M 2$ e o papel moeda em poder do público em relação a $\mathrm{M} 2$ e em relação ao $\mathrm{PIB}$, a razão entre o crédito bancário direcionado ao setor privado e o PIB, a divisão entre o crédito total do sistema financeiro direcionado ao setor privado pelo PIB 
e, por último, a proporção de M2 no PIB. O autor realiza seus testes de causalidade considerando um conjunto de séries temporais anuais entre 1947 e 2000, ou com séries que começam um pouco mais tarde, dependendo da disponibilidade de informações sobre o crédito direcionado ao setor privado.

Marques Jr. e Porto Jr. (2004) usam séries de tempo brasileiras anuais de 1950 a 2000. Os autores procuram qualificar o sistema financeiro brasileiro por meio de indicadores para o sistema bancário e para o mercado de capitais, dando uma dimensão de seus tamanhos, níveis de atividade e graus de eficiência. Para captar o efeito tamanho do sistema bancário e de capitais, é utilizada, respectivamente, a soma dos depósitos bancários à vista e a prazo em relação ao PIB e o valor das transações em bolsa dividido pelo PIB. O nível de atividade nesses mercados é representado pela razão entre o crédito bancário direcionado ao setor privado em relação ao PIB e o valor anual das transações no mercado de capitais como proporção do PIB. Por fim, a eficiência bancária e do mercado de capitais é aproximada, na sequência, pela razão entre o crédito direcionado ao setor privado e o PIB e pela soma dos depósitos bancários à vista e a prazo, dividida pelo PIB e pelo valor anual das transações em bolsa sobre o valor total da capitalização das ações negociadas. Embora haja alguma variação, dependendo da proxy utilizada para representar o sistema financeiro, os resultados reportados nesses trabalhos mostram ampla evidência de uma relação unidirecional do sistema financeiro para crescimento econômico, em consonância com a literatura teórica apresentada anteriormente.

Pires (2005), seguindo a estratégia proposta em King e Levine (1993b), analisa o impacto do crédito sobre o crescimento econômico nos municípios brasileiros. As informações utilizadas constituem um painel com dados para os anos de 1991 e 2000. Como variáveis financeiras, são inclusas a poupança, depósitos à vista, depósitos a prazo do setor privado e aplicações financeiras. As evidências apontam que o sistema financeiro possui relevância no processo de desenvolvimento dos municípios no país. No entanto, os municípios do Sudeste parecem se beneficiar mais desses resultados do que aqueles das demais regiões. Segundo Pires (2005), tal evidência pode estar atrelada ao fato de que talvez exista um nível mínimo de desenvolvimento a partir do qual o sistema financeiro possa contribuir com o crescimento econômico. Para testar tal hipótese, o autor estimou equações agregando os municípios conforme os grupos de renda inicial. Os resultados apontaram que os municípios mais pobres não exibem re- 
lação crédito e crescimento o que, conforme o autor, sugere ausência de demanda por crédito em tais localidades.

Reichstul e Lima (2006) avaliaram a causalidade entre crédito e nível de atividade econômica na região metropolitana do município de São Paulo para o período compreendido entre janeiro de 1993 e dezembro de 2003. Os autores estimam um modelo de Vetores Autorregressivos (VAR) bivariado tendo como variáveis endógenas o Indicador de Movimentação Econômica (IMEC), calculado pela FIPE, como proxy para atividade econômica, e distintos indicadores de crédito: crédito total, financiamentos, empréstimos e títulos descontados e desembolsos do BNDES. O modelo conta, ainda, com três variáveis exógenas em sua especificação: juros reais, desvio padrão mensal dos retornos diários do IBOVESPA e dummies de tempo. Os resultados dos testes de causalidade de Granger sugerem que existe causalidade em ambas as direções, para três das séries de crédito total e financiamentos. Os testes para a série de empréstimos e títulos descontados apresentaram bicausalidade somente para o período compreendido até outubro de 1997. Para a amostra compreendida após esse período, os testes não apresentaram causalidade em nenhuma direção. Também os testes que consideram a série desembolsos do BNDES não apresentaram resultados significativos em nenhuma direção.

Araújo e Dias (2006), através de um modelo VAR e de testes de exogeneidade, analisam como as variáveis monetárias afetam o crescimento. Foram utilizadas séries trimestrais para o período compreendido entre o primeiro trimestre de 1980 e o terceiro trimestre de 2003 com as seguintes informações: taxas de juros, medida pela Selic nominal; reservas bancárias, medida pela razão depósito compulsório/base monetária; imposto inflacionário, calculado pelo Ipea; e crescimento, medido pela taxa de crescimento do PIB. Os resultados dos testes apresentam a taxa de juros e o imposto inflacionário como variáveis endógenas ao passo que as reservas bancárias se apresentaram exógenas. Vale ressaltar que, a partir dos modelos estimados, concluiu-se que as taxas de juros e o imposto inflacionário afetam negativamente a variabilidade do crescimento não possuindo, portanto, efeitos de longo prazo. Por outro lado, elevações das reservas bancárias apresentaram reduções permanentes na taxa de crescimento da economia.

Kroth e Dias (2006) verificam a contribuição do crédito bancário e do capital humano na determinação do crescimento econômico utilizando um painel de municípios brasileiros no período compreendido entre 1999 
e 2003. Os resultados indicam causalidade das operações de crédito e capital humano sobre o crescimento econômico dos municípios no período analisado. Como medida de crescimento, empregou-se a taxa de variação do PIB municipal ao passo que para as informações de crédito foram utilizadas as seguintes variáveis: operações de crédito, poupança e número e agências. Nos diversos modelos estimados, apenas as operações de crédito se mostraram robustas em explicar o crescimento dos municípios.

Rocha e Nakane (2007) procuram verificar, com uma análise de painel para as unidades da Federação, a direção da causalidade entre desenvolvimento econômico e indicadores financeiros para o Brasil. Entre os indicadores financeiros, foram selecionadas as seguintes informações: o volume real de crédito bancário por agência em cada estado e o volume real de depósitos bancários totais (à vista e a prazo) por agência em cada estado. O período analisado se estende de 1995 a 2002. Os autores empregam um procedimento de teste baseado nas metodologias sugeridas por Holtz-Eakin, Newey e Rosen (1988) e Granger e Huang (1997). Os resultados encontrados evidenciam que os indicadores financeiros utilizados Granger-causam produto. Posteriormente, um segundo resultado utilizando dados mensais compreendidos entre os meses de fevereiro de 1997 e agosto de 2003 é apresentado. Nesta segunda estratégia de modelagem, verifica-se a causalidade em sentido contrário, ou seja, o produto Granger-causa sistema financeiro. Segundo Rocha e Nakane (2007), essa inversão de resultados pode ser explicada pela natureza de longo prazo dos fenômenos por trás da relação entre sistema financeiro e produto, ao passo que, considerando o curto prazo, o sistema financeiro acaba por se tornar um reflexo das condições reais da economia.

Missio, Jayme Jr. e Oliveira (2010) também fazem uma análise para as unidades da Federação por meio de um painel com o emprego de regressões quantílicas. Para mensurar o desenvolvimento do sistema financeiro, os autores seguiram duas estratégias: a primeira baseada em King e Levine (1993b) e a segunda baseada em Marques Jr. e Porto Jr. (2004). Os indicadores financeiros utilizados neste estudo são: passivo exigível (soma dos depósitos à vista e a prazo) sobre PIB, crédito do sistema financeiro sobre o PIB e crédito total sobre depósitos totais. Os autores empregaram, ainda, indicadores que buscavam verificar a preferência pela liquidez dos bancos (PLB) e preferência pela liquidez do público. O primeiro foi construído dividindo-se o total de depósitos à vista sobre o total das operações de 
crédito, ao passo que para o segundo foi empregada a razão entre os depósitos à vista e os depósitos totais. As evidências encontradas pelos autores apontam para uma relação positiva entre desenvolvimento do sistema financeiro e crescimento econômico. ${ }^{5}$

Alguns trabalhos recentes têm argumentado que a relação entre sistema financeiro e crescimento econômico é dependente das características das economias analisadas (Demetriades; Hussein, 1996; Luintel; Khan, 1999; Odedokun, 1996) e do horizonte de tempo analisado (King; Levine, 1993a). O presente artigo se encontra dentro desta segunda perspectiva, empregando uma metodologia que permite um avanço em relação à classe de testes de causalidade encontrados na literatura, uma vez que o domínio da frequência permite avaliar a causalidade de Granger nos ciclos de diferentes frequências.

Primeiramente, procedimentos com séries de tempo no domínio da frequência são relativamente pouco empregados na literatura econômica. Justifica-se o uso de tal metodologia baseado no fato de que o uso de técnicas espectrais permite decompor toda a variabilidade das séries envolvidas em ciclos com distintas frequências. Assim, é possível verificar se séries exibem causalidade a partir de correlações de curto prazo (alta frequência), longo prazo (baixa frequência) ou ambas. Nesse sentido, o artigo contribui ao utilizar uma metodologia de teste para o domínio da frequência, mas que é estimada integralmente a partir de modelos tradicionais de vetores autorregressivos, sendo, para o nosso conhecimento, a primeira aplicação deste tipo de procedimento para a análise da relação causal entre sistema financeiro e crescimento econômico para o caso brasileiro. Na literatura internacional, poucos trabalhos utilizaram tal metodologia até então (Ramirez, 2013; Gómez-González et al., 2014; Stolbov, 2015), mas ainda sem aplicações para o Brasil.

De forma mais relevante, com a metodologia apresentada na sequência, podemos selecionar algumas frequências, equivalentes a ciclos de diferentes períodos, para avaliarmos a causalidade-Granger. Como os resultados mostram, esse procedimento traz novas informações ao debate, as quais não podem ser obtidas nos testes de causalidade usuais com séries de tempo no domínio do tempo.

5 Recentemente, alguns artigos têm destacado os efeitos regionalizados da atividade bancária como resultado das diferentes estratégias das instituições financeiras em distintas regiões (Crocco; Figueiredo; Santos, 2010; Crocco; Santos; Amaral, 2010). 


\section{Metodologia}

Breitung e Candelon (2006) propõem um procedimento simples para a realização de testes de causalidade de Granger no domínio da frequência. A análise de séries de tempo no domínio da frequência é o análogo da tradicional abordagem no domínio do tempo, tendo como referência a decomposição da variável de interesse em ciclos de diferentes frequências. ${ }^{6}$

$\mathrm{Na}$ prática, essa abordagem oferece flexibilidade para estimação da relação econométrica de interesse em diferentes pontos do espectro de frequências. No caso do exame da causalidade entre duas variáveis $x_{t}$ e $y_{t}$, é possível avaliar se a precedência temporal altera-se, dependendo da frequência $\omega$ do ciclo considerada. Nos termos do problema estudado neste trabalho, seria o sistema financeiro um fator causal de crescimento econômico? Mas apenas para ciclos de frequência baixa (longo prazo)? Ou seria tal causalidade observada apenas em frequências mais altas, típicas dos ciclos de negócios de curto prazo?

Para compreender a natureza do teste proposto por Breitung e Candelon (2006), considere, inicialmente, a representação autorregressiva para o vetor $z_{t}=\left[y_{t}, x_{t}\right]^{\prime}$, com $\mathrm{t}=1 \ldots \mathrm{T}$ :

$$
\Theta(L) z_{t}=e_{t}
$$

em que $\Theta(L)=I-\Theta_{1} L-\Theta_{2} L^{2}-\ldots-\Theta_{p} L^{p}$ é um polinômio $2 \times 2$, com $L^{k} g_{t}=$ $=g_{t-k}$. Além disso, $E\left(e_{t}\right)=0$ e $E\left(e_{t} e_{t}^{\prime}\right)=\Sigma$. Seja G é uma matriz triangular inferior que faz a decomposição de Cholesky do VAR definido em (1), com $G^{\prime} G=\Sigma^{-1}, \eta_{t}=G e_{t}$ e $E\left(\eta_{t} \eta_{t}^{\prime}\right)=I$.

Assumindo estacionariedade para o vetor $z_{t}$, podemos escrever (1) em sua forma MA:

$$
\begin{aligned}
& z_{t}=\Phi(L) e_{t}=\left[\begin{array}{ll}
\Phi_{11}(L) & \Phi_{12}(L) \\
\Phi_{21}(L) & \Phi_{22}(L)
\end{array}\right] \times\left[\begin{array}{l}
e_{1 t} \\
e_{2 t}
\end{array}\right], \text { ou } \\
& z_{t}=\Psi(L) \eta_{t}=\left[\begin{array}{ll}
\Psi_{11}(L) & \Psi_{12}(L) \\
\Psi_{21}(L) & \Psi_{22}(L)
\end{array}\right] \times\left[\begin{array}{l}
\eta_{1 t} \\
\eta_{2 t}
\end{array}\right]
\end{aligned}
$$

6 As análises de séries de tempo no domínio do tempo e no domínio da frequência não são excludentes. Toda série temporal covariância-estacionária possui representação em ambos os domínios de análise. Para uma apresentação formal da análise de séries de tempo no domínio da frequência, ver Hamilton (1994) e Brillinger (1981). 
em que $\Phi(L)=\Theta(L)^{-1}$ e $\Psi(L)=\Phi(L) G^{-1}$.

$\mathrm{Na}$ definição consagrada por Granger (1969), a causalidade $x_{t} \Rightarrow y_{t}$ é avaliada por meio da comparação entre as especificações alternativas (i) e (ii) para a equação de $y_{t}$ na expressão (2):
(i) $y_{t}=\Psi_{21}(L) \eta_{1 t}+\Psi_{22}(L) \eta_{2 t}$
(ii) $y_{t}=\Psi_{22}(L) \eta_{2 t}$

Se o poder explicativo de (i) e (ii) são semelhantes, diz-se que $x_{t}$ não Granger-causa $y_{t}$.

No domínio da frequência, medida semelhante para a causalidade pode ser considerada. Em primeiro lugar, deve-se tomar a representação espectral para $y_{t}$ na expressão (2):

$$
f_{y}(\omega)=\frac{1}{2 \pi}\left\{\left|\Psi_{21} e^{-i \omega}\right|^{2}+\left|\Psi_{22} e^{-i \omega}\right|^{2}\right\}
$$

A expressão (3) reporta a função de densidade espectral da variável $y_{t}$, construída a partir da formulação MA do processo estocástico (1). Grosso modo, para cada $\omega$, a densidade espectral revela a porção da variância do processo $y_{t}$ que é devida aos componentes periódicos (seno e coseno) na frequência associada.

No presente caso, a densidade espectral de $y_{t}$ é dada pela composição da fração da variância associada às defasagens de $y_{t}$ e $x_{t}:\left|\Psi_{21} e^{-i \omega}\right|^{2}$ e $\left|\Psi_{22} e^{-i \omega}\right|^{2}$, respectivamente. Com isso, é fácil ver que um teste de causalidade pode ser obtido pela comparação da variância devida ao modelo irrestrito, $f_{y}(w)$, e o processo em que a parcela devida a $x_{t}$ não é considerada (Geweke, 1982; Hosoya, 1991):

$$
M_{x \Rightarrow y}(\omega)=\log \left[\frac{2 \pi f_{y}(\omega)}{\left|\Psi_{22} e^{-i \omega}\right|^{2}}\right]=\log \left[1+\frac{\left|\Psi_{21} e^{-i \omega}\right|^{2}}{\left|\Psi_{22} e^{-i \omega}\right|^{2}}\right]
$$

A medida de causalidade (4) será zero se $\left|\Psi_{21} e^{-i \omega}\right|^{2}=0$, indicando que $x_{t}$ não Granger-causa $y_{t}$ na frequência $\omega$. Partindo dessa condição, Breitung e Candelon (2006) propõem um procedimento simples para avaliação desta hipótese de causalidade, usando estimativas tradicionais feitas no domínio do tempo. 
Como $\Psi(L)=\Phi(L) G^{-1}$, temos que $\left|\Psi_{21} e^{-i \omega}\right|^{2}=0$ se $\left|\Theta_{21} e^{-i \omega}\right|^{2}=0$. Por outro lado, podemos reescrever $\left|\Theta_{21} e^{-i \omega}\right|^{2} \operatorname{como}\left|\Theta_{21} e^{-i \omega}\right|^{2}=\mid \sum_{j=1}^{p} \theta_{21, j} \cos (j \omega)-$ $-\sum_{j=1}^{p} \theta_{21, j} \operatorname{sen}(j \omega) i$, onde $\theta_{21, j}$ é o elemento $(2,1)$ associado à j-ésima defasagem de $\Theta(L)$, estimado no VAR proposto em (1).

Assim, uma condição necessária e suficiente para $M_{x \Rightarrow y}(\omega)=0$ é o teste conjunto das restrições:

$$
\left\{\begin{array}{l}
\sum_{j=1}^{p} \theta_{21, j} \cos (j \omega)=0 \\
\sum_{j=1}^{p} \theta_{21, j} \operatorname{sen}(j \omega)=0
\end{array}\right.
$$

Na prática, o procedimento consiste da estimação do VAR representado na expressão (1) e o posterior teste do conjunto de restrições acima. Neste trabalho, a estatística de teste da causalidade de $x_{t}$ para $y_{t}$ na frequência $\omega$ será a Norma Euclidiana para o vetor formado pelas restrições (5). Sob a hipótese de não-causalidade, o conjunto de restrições formará um vetor que, necessariamente, terá norma igual a zero. Portanto, o teste causalidade torna-se:

$$
\left\{\begin{array}{l}
H_{0}: R(\omega)=0 \\
H_{A}: R(\omega) \neq 0
\end{array}\right.
$$

onde $R(\omega)=\sqrt{\left(\sum_{j=1}^{p} \theta_{21, j} \cos (j \omega)\right)^{2}+\left(\sum_{j=1}^{p} \theta_{21, j} \operatorname{sen}(j \omega)\right)^{2}}$, que se distribui assintoticamente como uma qui-quadrado com um grau de liberdade. É importante notar que a estatística de teste proposta é função de $\omega$, o que permite a realização do teste de causalidade para ciclos em diferentes frequências. $^{7}$

7 Serão estimadas $j$ frequências de Fourier $\omega_{j}=\frac{2 j \pi}{T}, \operatorname{com} \mathrm{j}=1, \ldots, \mathrm{T} / 2$. Cada $\omega_{j}$ define um período de ciclo $P_{j}=\frac{2 \pi}{\omega_{j}}$, em que baixas (altas) frequências estão associadas a ciclos de período longo (curto). Para mais detalhes, ver Hamilton (1994). 


\section{Resultados}

Os testes de causalidade serão realizados com base nas informações do World Development Indicators \& Global Development Finance do Banco Mun$\mathrm{dial}^{8}$ para os anos entre 1960 e 2010. Seguindo a literatura aplicada, para o lado financeiro, selecionamos a variável crédito ao setor privado como proporção do PIB (CRED). Do lado real, a variável usada foi o PIB per capita em dólares constantes de 2000 (PIB). O uso da base de dados do Banco Mundial tem a vantagem de ampliar a extensão tempora $1^{9}$ e, ainda, permitir maior comparabilidade com a literatura internacional. ${ }^{10}$

Neste ponto, valem alguns comentários acerca da utilização da variável CRED como representação para o lado financeiro. Conforme a conceituação apresentada na seção 2 , o tratamento dos problemas de informação e a consequente redução do custo de financiamento externo, por meio das cinco funções descritas, representam a ligação do sistema financeiro com crescimento econômico. Com isso, seguindo Levine (2005), podemos definir desenvolvimento financeiro como uma melhora na capacidade de exercer estas funções, o que representa um desafio do ponto de vista de representação empírica. Logo, implicitamente, adotamos a hipótese de que a variável CRED, em alguma medida, permite captar este mecanismo de melhora no funcionamento do sistema financeiro que reduz o prêmio de financiamento externo.

Assim, o vetor de interesse, inicialmente, será dado por $z_{t}=\left[P I B_{t} C R E D_{t}\right]$. Como as representações espectrais requerem estacionariedade, as variáveis são modeladas em logaritmo natural e na primeira diferença. Os gráficos destas séries podem ser vistos no apêndice (Figura A1).

A série temporal para crédito no Brasil exibe uma queda acentuada em 1990, representando a forte contração ocorrida entre 1989 e 1990. Entretanto, o crédito retoma seu nível em 1993 para sofrer nova contração no

8 Disponível em http://databank.worldbank.org.

9 Para mensurar o nível de crédito, por exemplo, o Banco Central do Brasil disponibiliza diversas medidas com desagregações variadas. A extensão temporal, entretanto, é bastante pequena, sendo algumas séries iniciadas apenas nos anos 2000. O emprego de um intervalo de tempo mais longo, ainda que com dados em menor frequência, é preferível em nosso caso, uma vez que buscamos examinar relações temporais em componentes cíclicos de curto e longo prazo. Campbell e Perron (1991), por exemplo, destacam as vantagens de maiores horizontes temporais sobre o poder de testes tradicionais de séries de tempo.

10 Esta fonte de dados é bastante empregada pela literatura relacionada, por exemplo, Arcand, Berkes e Panizza (2012) e Calderon e Liu (2003). 
ano seguinte. A forte expansão recente do crédito é ilustrada pelas taxas de crescimento elevadas desta variável ao longo dos anos 2000. Já a série de crescimento econômico revela alguns importantes episódios recentes da economia brasileira, como o período de forte crescimento nos anos 70 e os períodos de estagnação nos anos 80 e na recente crise financeira internacional em 2008.

A decomposição das variâncias do PIB e do crédito ao longo das frequências pode ser visualizada a partir dos periodogramas das séries (Figuras A2 e A3). ${ }^{11}$ Uma característica interessante é o fato de a variabilidade de longo prazo do crédito ser pouco relevante. $O$ periodograma apresenta seu pico na frequência $\omega=1.29$. Isso significa que ciclos de aproximadamente cinco anos correspondem à maior parte da variabilidade do crédito no país. Na série de $\mathrm{PIB}$, predomina o padrão típico de espectros de séries econômicas de maior concentração da variância em frequências mais baixas (Granger, 1966).

A partir do exposto, torna-se interessante avaliar como se comporta a correlação entre as séries de PIB e crédito nas diversas frequências. Assim, apresentamos a coerência ao quadrado, que, grosso modo, ${ }^{12}$ decompõe a correlação entre duas séries distintas dentro das frequências de Fourier (Figura A4). Vemos que as séries apresentam correlação predominante na baixa frequência, significando, assim, que ciclos de longo prazo dessas variáveis estariam mais correlacionados do que no curto prazo.

O primeiro passo para a realização dos testes de causalidade é a estimativa do VAR para $z_{t}$. Para seleção da ordem do modelo VAR, optou-se pela utilização de dois critérios: (i) ausência de autocorrelação de $1^{\text {a }}$ ordem dos resíduos, segundo a estatística LM para o teste de Breusch (1978) e Godfrey (1978), e (ii) minimização do critério AIC. A tabela A1 no Apêndice reporta as estatísticas de testes para as diferentes especificações. Seguindo os critérios apontados, estimamos um modelo $\operatorname{VAR}(4)$ para $z_{t}=\left[P I B_{t} C R E D_{t}\right]$;, de onde foram obtidos os parâmetros empregados na construção da estatística para o teste (5). ${ }^{13}$

11 O periodograma é a contrapartida amostral para a função de densidade espectral teórica de uma série qualquer. Os periodogramas foram estimados com uma suavização por janela retangular de tamanho 8. Para mais detalhes, ver Hamilton (1994).

12 O espectro de coerência (ou coerência ao quadrado) é uma estatística calculada a partir do espectro conjunto de duas ou mais variáveis, dando dimensão da importância relativa dos ciclos em comum que as variáveis analisadas possuem em uma determinada frequência $\omega$. Ver Hamilton (1994), para uma discussão mais detalhada.

13 Vale mencionar que, além da indicação dada pelos critérios estatísticos, é intuitivo supor que a relação dinâmica envolvendo sistema financeiro e crescimento econômico requeira 
A tabela 1 traz as estatísticas de teste e os respectivos p-valores para a avaliação da hipótese nula de não causalidade entre sistema financeiro e crescimento econômico em algumas frequências selecionadas. A tabela reporta, ainda, o período de ciclo associado a cada frequência. Por exemplo, a frequência mais baixa $(\omega=0.16)$ está associada a ciclos com período de 40 anos, o mais longo identificado por nossos dados. No outro extremo, os ciclos de prazo mais curto (2 anos) estão relacionados com a frequência mais elevada $(\omega=3.14)$.

Os resultados mostram que há variação nos resultados dos testes de causalidade, dependendo da frequência considerada, o que mostra a relevância do procedimento adotado neste trabalho. Por um lado, em nenhuma das frequências testadas há sinais de causalidade entre crescimento econômico e crédito. Porém, na direção contrária, há evidências de causalidade de crédito para crescimento, mas apenas nas frequências mais baixas, ou seja, no longo prazo.

Tabela 1 Testes de causalidade para frequências selecionadas

\begin{tabular}{lrr|r|r|r|r|r}
\hline Frequência $\left(\omega_{j}\right)$ & $\mathbf{0 . 1 6}$ & $\mathbf{0 . 3 1}$ & $\mathbf{0 . 6 3}$ & $\mathbf{1 . 2 6}$ & $\mathbf{1 . 5 7}$ & $\mathbf{3 . 1 4}$ \\
\hline Período (anos) & $\mathbf{4 0}$ & $\mathbf{2 0}$ & $\mathbf{1 0}$ & $\mathbf{5}$ & $\mathbf{4}$ & $\mathbf{2}$ \\
\hline CRED $\Rightarrow$ PIB & ${ }^{* * *} 10.94$ & $* * * 10.87$ & $* * * 10.3$ & 1.15 & 2.05 & 0.75 \\
& $(0.00)$ & $(0.00)$ & $(0.00)$ & $(0.28)$ & $(0.15)$ & $(0.39)$ \\
\hline PIB $\Rightarrow$ CRED & 0.10 & 0.18 & 0.27 & 0.35 & 0.43 & 0.09 \\
& $(0.75)$ & $(0.67)$ & $(0.60)$ & $(0.55)$ & $(0.51)$ & $(0.76)$ \\
\hline
\end{tabular}

Nota: P-valor entre parênteses.

$*^{*},{ }^{* *},{ }^{* *}$ denotam significância estatística a 10\%, 5\% e 1\%, respectivamente.

Fonte: Elaborado pelos autores.

A figura 1 permite uma avaliação visual da relação de causalidade entre sistema financeiro e crescimento ao longo de todas as frequências no espectro $(0$, $\pi)$. A linha vermelha representa a estatística do teste de não-causalidade de PIB para Crédito, ao passo que a linha cinza testa a causalidade no sentido contrário. A linha escura, por outro lado, delimita a região de aceitação do teste, reportando o valor crítico para a distribuição qui-quadrado com 1 grau

uma estrutura de defasagens superior a um ano. Na hipótese de causalidade na direção de crescimento econômico para desenvolvimento financeiro, é possível que os efeitos reais sobre o setor financeiro se estendam por vários períodos de tempo, em termos de volumes transacionados ou na criação de novos produtos. Na direção contrária, os possíveis efeitos do sistema financeiro sobre fatores reais, como a alocação de capital e nível de investimento, também devem se dar de forma defasada no tempo. 
de liberdade e 5\% de significância. É interessante notar que a figura parece indicar significância estatística para a causalidade de crédito para PIB em ciclos de frequências próximas a 3 anos (ou $0,7 \pi$ ), algo que não é visualizado na Tabela 1. Para frequências mais altas, não há evidências de causalidade.

Figura 1 Estatística de testes de causalidade e frequências de ciclos $(0, \pi)$

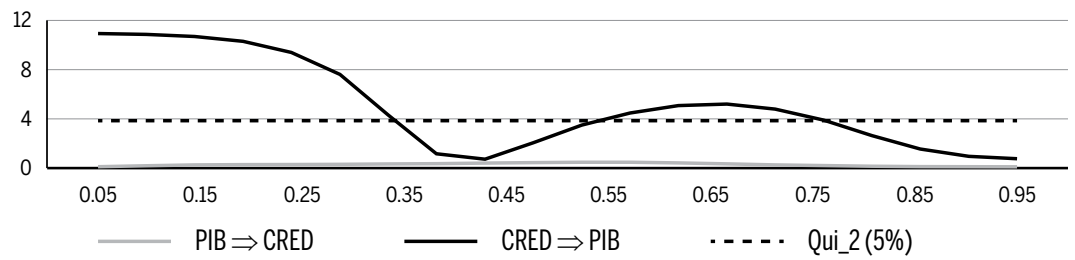

Fonte: Elaborado pelos autores.

Modelos bivariados podem resultar em inferências errôneas a respeito de causalidades, caso variáveis relevantes sejam omitidas (Lutkepohl, 1982). No caso da relação envolvendo sistema financeiro e crescimento econômico, é possível que fatores ligados à produtividade e tecnologia impactem a alocação de fatores com reflexos no crescimento econômico. Em razão disso, visando analisar a robustez dos resultados, repetiremos os procedimentos acima, incluindo o controle pela acumulação do fator de produção capital. A variável de controle incluída refere-se à primeira diferença do logaritmo natural da formação bruta de capital fixo como proporção do PIB $(F B K F)$, também obtida no Banco Mundial. Os modelos seguem as mesmas especificações anteriores, a não ser pela inclusão da variável $F B K F$. A tabela 2 reporta os resultados dos novos testes.

Tabela 2 Testes de causalidade para frequências selecionadas, controle por $F B K F$

\begin{tabular}{|c|c|c|c|c|c|c|}
\hline$\omega_{j}$ & 0.16 & 0.31 & 0.63 & 1.26 & 1.57 & 3.14 \\
\hline Período & 40 & 20 & 10 & 5 & 4 & 2 \\
\hline \multirow[t]{2}{*}{ CRED $\Rightarrow \mathrm{PIB}$} & $* * * 12.03$ & $* * * 12.12$ & $* * * 12.3$ & 2.36 & 0.74 & 0.73 \\
\hline & $(0.00)$ & $(0.00)$ & $(0.00)$ & $(0.12)$ & $(0.39)$ & (0.39) \\
\hline \multirow[t]{2}{*}{$\mathrm{PIB} \Rightarrow$ CRED } & 0.13 & 0.48 & 0.60 & 0.68 & 0.66 & 0.00 \\
\hline & $(0.72)$ & $(0.49)$ & $(0.44)$ & $(0.41)$ & $(0.42)$ & $(1.00)$ \\
\hline
\end{tabular}

Nota: P-valor entre parênteses.

* , **, *** denotam significância estatística a 10\%, 5\% e 1\%, respectivamente.

Fonte: Elaborado pelos autores. 
Como podemos ver, as conclusões acerca da direção das causalidades se repetem. A evidência de causalidade de sistema financeiro para crescimento econômico no Brasil encontra respaldo em trabalhos anteriores (por exemplo, Marques Jr.; Porto Jr., 2004; Matos, 2002; Rocha; Nakane, 2007; entre outros). Rocha e Nakane (2007), apesar de não apresentarem um teste formal, argumentam que a causalidade entre crédito e PIB estaria associada a fatores de longo prazo. Neste trabalho, com o uso de técnicas para séries temporais no domínio da frequência, podemos lançar luz sobre esta hipótese, fornecendo evidências favoráveis à causalidade entre crédito e crescimento econômico em ciclos de longo prazo.

\section{Conclusões}

O presente trabalho teve como objetivo apresentar testes de causalidade entre sistema financeiro e crescimento econômico para o Brasil. A principal contribuição para a literatura é a aplicação dos procedimentos de teste para o domínio da frequência, desenvolvidos por Breitung e Candelon (2006). A vantagem da abordagem no domínio da frequência é a possibilidade de decomposição dos testes em ciclos de diferentes frequências.

Os resultados explicitam a complexidade da relação envolvendo sistema financeiro e crescimento econômico. Conforme Arestis e Demetriades (1997), a natureza da relação parece específica a cada país. Mesmo para um determinado país, os dados mostram que a direção da causalidade depende do prazo do ciclo considerado. Ou seja, a abertura da análise no domínio da frequência parece importante para o exame da relação entre sistema financeiro e crescimento econômico.

Para o Brasil, a causalidade de sistema financeiro para crescimento econômico se sustenta de forma robusta. Tal evidência de causalidade, contudo, é observada apenas nos ciclos de longo prazo. Por outro lado, na direção contrária, em nenhuma das frequências testadas há sinais de causalidade entre crescimento econômico e crédito.

\section{Referências}

ARCAND, J. L.; BERKES, E.; PANIZZA, U. Too much finance?. IMF Working Paper. WP12/ 161, 2012. 
ARAÚJO, E. C.; DIAS, J. Endogeneidade do setor financeiro e crescimento econômico: uma análise empírica para a economia brasileira (1980-2003). Revista de Economia Contemporânea, 10, p. 575-609, 2006.

ARESTIS, P.; DEMETRIADES, P. Financial development and economic growth: Assessing the evidence. The Economic Journal, 107, p. 783-799, 1997.

BARRO, R.; SALA-I-MARTIN, X. Economic Growth. Boston: The MIT Press, 2004.

BECK, T.; LEVINE, R.; LOAYZA, N. Finance and sources of growth. Journal of Financial Economics, 58, p. 261-300, 2000.

BERNANKE, B. Non monetary effects of financial crisis in propagation of Great Depression. American Economic Review, 73, p. 257-276, 1983.

BREITUNG, J.; CANDELON, B. Testing for short- and long-run causality: A frequency-domain approach. Journal of Econometrics, 132, p. 363-378, 2006.

BREUSCH, T. Testing for autocorrelation in dynamic linear models. Australian Economic Papers, 17, p. 334-355, 1978.

BRILLINGER, D. Time series: Data analysis and theory. San Francisco: Holden-Day Inc, 1981.

CAMPBELL, J. Y.; PERRON, P. Pitfalls and opportunities: what macroeconomists should know about unit roots. In: BLANCHARD, O.; FISCHER, S. (Eds.). NBER Macroeconomics Annual. Cambridge, MA: MIT Press, p. 141-201, 1991.

CALDERÓN, C.; LIU, L. The direction of causality between financial development and economic growth. Journal of Development Economics, 72, p. 321-334, 2003.

CROCCO, M.; FIGUEIREDO, A.; SANTOS, F. Differentiated banking strategies across the territory: an exploratory analysis. Journal of Post Keynesian Economics, 33, p. 127-150, 2010.

CROCCO, M; SANTOS, F. e AMARAL, P. The Spatial Structure of Financial Development in Brazil. Spatial Economic Analysis, 5, p. 181-203, 2010.

DEMETRIADES, P.; HUSSEIN, K. Does financial development cause economic growth? Time series evidence from 16 countries. Journal of Development Economics, 51, p. 387-411, 1996.

GEWEKE, J. Measurement of linear dependence and feedback between multiple time series. Journal of American Statistical Association, 77, p. 304-324, 1982.

GODFREY, L. Testing against general autoregressive and moving average error when the regressors Include lagged dependent variables. Econometrica, 46, p. 1293-1302, 1978.

GOLDSMITH, R.W. Financial Structure and Development. New Haven. Conn.: Yale University Press, 1969.

GÓMEZ-GONZÁLEZ, J.; OJEDA-JOYA, J.; ZÁRATE, H.; TENJO-GALARZA, F. Testing for causality between credit and real business cycles in the frequency domain: an illustration. Applied Economics Letters, 21, p. 697-701, 2014.

Granger, C. The typical spectral shape of an economic variable. Econometrica, 34, p. 150-161, 1966.

GRANGER, C. Investigating causal relations by econometric models and cross-spectral methods. Econometrica, 37, p. 424-438, 1969.

GRANGER, C.; HUANG, L. Evaluation of Panel Data Models: Some suggestions from Time Series. Mimeo. U.C. San Diego, p. 1-29, 1997. 
GURLEY, J. G.; SHAW, E. Financial aspects of economic development. American Economic Review, 45, p. 515-538, 1955.

HAMILTON, J. Time series analysis. Princeton: Princeton University Press, 1994.

HOLTZ-EAKIN, D.; NEWEY, W.; ROSEN, H. Estimating Vector Autoregressions with panel data. Econometrica, 56, p. 1371-1395, 1988.

HOSOYA, Y. The decomposition and measurement of interdependence between secondorder stationary process. Probability Theory and Related Fields, 88, p. 429-444, 1991.

JOHANSEN, S. Statistical analysis of cointegration vectors. Journal of Economic Dynamic and Control, 12, p. 231-254, 1988.

KING, R.; LEVINE, R. Financial intermediation and economic development. In: MAYER, C.; VIVES, X. (Eds.). Capital Markets and Financial Intermediation. Cambridge: Cambridge University Press, 1993a.

KING, R.; LEVINE, R. Finance and growth: Schumpeter might be right. The Quarterly Journal of Economics, 108, p. 717-739, 1993 b.

KROTH, D.; DIAS, J. A contribuição do crédito bancário e do capital humano no crescimento econômico dos municípios brasileiros: uma avaliação em painéis de dados dinâmicos. Anais do XXXIV Encontro Nacional de Economia da ANPEC - Associação Nacional dos Centros de Pós-Graduação em Economia, 2006.

LEVINE, R. Finance and growth: Theory and evidence. In: AGHION, P.; DURLAUF, S. (eds). Handbook of Economic Growth, v. 1, cap. 12. Elsevier. p. 865-34, 2005.

LEVINE, R.; LOAYZA, N.; BECK, T. Financial intermediation and growth: Causality and causes. Journal of Monetary Economics, 46, p. 31-77, 2000.

LUCAS, R. Jr. On the mechanism of economic development. Journal of Monetary Economics, 22, p. 3-42, 1988.

LUINTEL, K.; KHAN, M. A quantitative reassessment of the finance-growth nexus: evidence from a multivariate VAR. Journal of Development Economics, 60, p. 381-405, 1999.

LUTKEPOHL, H. Non-causality due to omitted variables. Journal of Econometrics, 19, p. 367378, 1982.

MARQUES JR., T. E.; PORTO JR., S.S. Desenvolvimento financeiro e crescimento econômico no Brasil - Uma avaliação econométrica. PPGE/UFRGS. Trabalho para Discussão 11, p. 1-20, 2004.

MATOS, O. C. Desenvolvimento do sistema financeiro e crescimento econômico no Brasil: Evidência de causalidade. Banco Central do Brasil. Trabalho para Discussão 49, p. 1-65, 2002.

MISSIO, F.; JAYME JR., F; OLIVEIRA, A. M. Desenvolvimento financeiro e crescimento econômico: teoria e evidência empírica para os estados brasileiros (1995-2004). CEDEPLAR UFMG. Texto para Discussão, n. 379, p. 1-34, 2010.

ODEDOKUN, M. Alternative econometric approaches for analyzing the role of the financial sector in economic growth: Time series evidence from LDCs. Journal of Development Economics, 50, p. 119-146, 1996.

PIRES, M. Crédito e crescimento econômico: evidências para os municípios brasileiros. Anais 
do VIII Encontro de Economia da Região Sul - ANPEC-SUL, Porto Alegre-RS, 2005.

RAJAN, R.; ZINGALES, L. Financial dependence and growth. American Economic Review, 88, p. $559-586,1998$.

RAMIREZ, F. The Relationship Between Credit and Business Cycles in Central America and the Dominican Republic. Banco Central de la República Dominicana. Documento de Trabajo, n. 2013-02, 2013.

REICHSTUL, D.; LIMA, G. Causalidade entre crédito bancário e nível de atividade econômica na região metropolitana de São Paulo: algumas evidências empíricas. Estudos Econômicos, 36, p. 779-801, 2006.

ROCHA, B. P; NAKANE, M. I. Sistema Financeiro e Desenvolvimento Econômico: Evidências de Causalidade em um Painel para o Brasil. Anais do XXXV Encontro Nacional de Economia - Associação Nacional dos Centros de Pós-Graduação em Economia, ANPEC. Recife, PE, 2007.

ROMER, P. Increasing returns and long-run growth. Journal of Political Economy, 94, p. $1002-$ 1037, 1986.

SOLOW, R. A contribution to the theory of economic growth. Quarterly Journal of Economics, 70, p. 65-74, 1956.

STIGLITZ, J.; WEISS, A. Credit rationing with imperfect information. American Economic Review, 71, p. 393-410, 1981.

STOLBOV, M. Causality between credit depth and economic growth: Evidence from 24 OECD countries. Bank of Finland. BOFIT Discussion Papers, n. 5, p. 1-38, 2015.

\section{Sobre os autores}

Bruno de Paula Rocha - rocha.b@ufabc.edu.br

Centro de Engenharia, Modelagem e Ciências Sociais Aplicadas da Universidade Federal do ABC (CECS/ UFABC).

São Bernardo do Campo, SP.

IgorViveiros de Souza - igorviveiros@cedeplar.ufmg.br

Universidade Federal de Minas Gerais. Belo Horizonte, MG.

\section{Sobre 0 artigo}

Recebido em 16 de dezembro de 2014. Aprovado em 12 de setembro de 2016. 


\section{APÊNDICE}

Tabela A1 Critérios de seleção do VAR

\begin{tabular}{l|r|r}
\hline Lag & AIC & LM $^{\mathbf{1}}$ \\
\hline $\mathbf{1}$ & -5.021 & $(0.00)$ \\
\hline $\mathbf{2}$ & -4.859 & $(0.04)$ \\
\hline $\mathbf{3}$ & -4.734 & $(0.02)$ \\
\hline $\mathbf{4}$ & -4.720 & $(0.08)$ \\
\hline $\mathbf{5}$ & -4.595 & $(0.26)$ \\
\hline $\mathbf{6}$ & -4.442 & $(0.22)$ \\
\hline
\end{tabular}

Nota: ${ }^{1}$ LM refere-se ao p-valor do teste de Breusch (1968) e Godfrey (1968) para autocorrelação de $1^{a}$ ordem dos resíduos do respectivo VAR.

Fonte: Elaborado pelos autores.

Figura A1 Séries de tempo

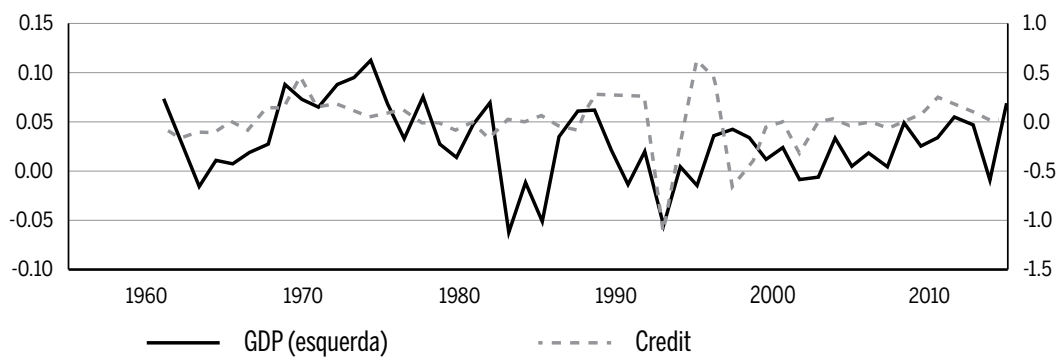

Time

Fonte: Elaborado pelos autores.

Figura A2 Periodograma suavizado do PIB

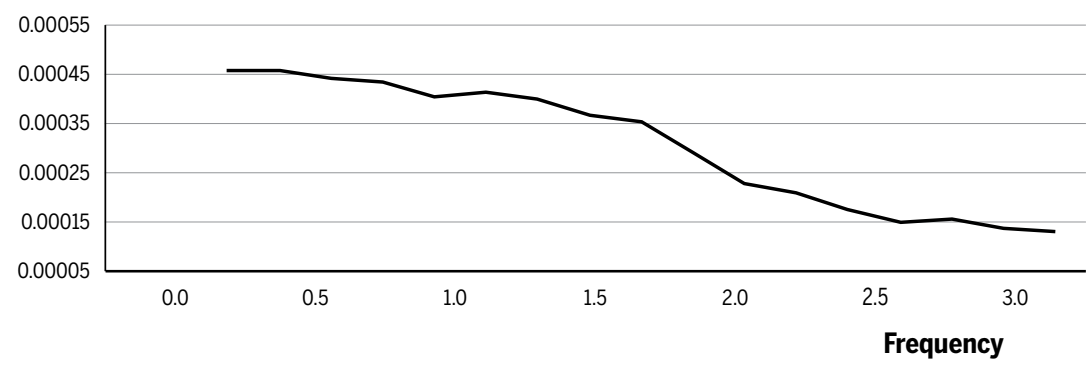

Fonte: Elaborado pelos autores. 
Figura A3 Periodograma suavizado do Crédito

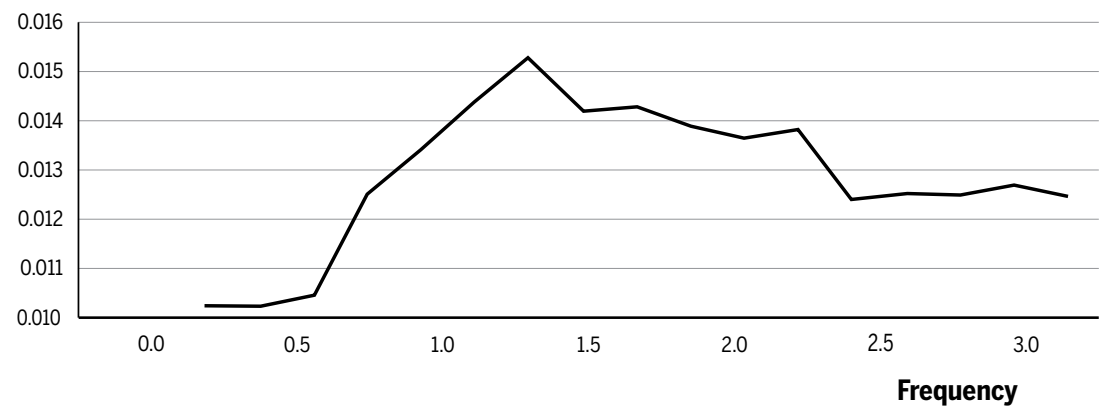

Fonte: Elaborado pelos autores.

Figura A4 Coerência ao quadrado entre Crédito e PIB

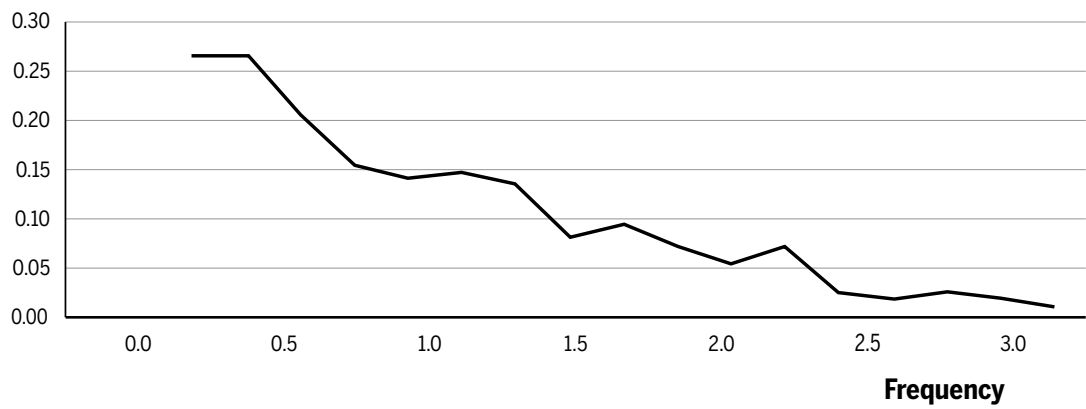

Fonte: Elaborado pelos autores. 